\title{
COSMETIC CROSSING CONJECTURE FOR GENUS ONE KNOTS WITH NON-TRIVIAL ALEXANDER POLYNOMIAL
}

\author{
TETSUYA ITO
}

\begin{abstract}
We prove the cosmetic crossing conjecture for genus one knots with non-trivial Alexander polynomial. We also prove the conjecture for genus one knots with trivial Alexander polynomial, under some additional assumptions.
\end{abstract}

\section{INTRODUCTION}

A cosmetic crossing is a non-nugatory crossing such that the crossing change at the crossing preserves the knot. A cosmetic crossing conjecture [Kir, Problem 1.58] asserts there are no such crossings.

Conjecture 1 (Cosmetic crossing conjecture). An oriented knot $K$ in $S^{3}$ does not have cosmetic crossings.

Here a crossing $c$ of a knot diagram $D$ is nugatory if there is a circle $C$ on the projection plane that transverse to the diagram $D$ only at $c$. Obviously the crossing change at a nugatory crossing always preserves the knot, so the cosmetic crossing conjecture can be rephrased that when a crossing change at a crossing $c$ preserves the knot, then $c$ is nugatory.

In BFKP] Balm-Friedl-Kalfagianni-Powell proved the following constraints for genus one knots to admit a cosmetic crossing.

Theorem 1.1. BFKP, Theorem 1.1, Theorem 5.1] Let $K$ be a genus one knot that admits a cosmetic crossing. Then $K$ has the following properties.

- $K$ is algebraically slice.

- For the double branched covering $\Sigma_{2}(K)$ of $K, H_{1}\left(\Sigma_{2}(K) ; \mathbb{Z}\right)$ is finite cyclic.

- If $K$ has a unique genus one Seifert surface, $\Delta_{K}(t)=1$.

In this paper, by using the 2-loop part of the Kontsevich invariant, we prove the cosmetic crossing conjecture for genus one knot with non-trivial Alexander polynomial.

Theorem 1.2. Let $K$ be a genus one knot. If $\Delta_{K}(t) \neq 1$, then $K$ satisfies the cosmetic crossing conjecture.

For genus one knot $K$ with $\Delta_{K}(t)=1$ we get an additional constraint for $K$ to admit a cosmetic crossing. Let $\lambda$ be the Casson invariant of integral homology spheres and let $w_{3}(K)=\frac{1}{36} V_{K}^{\prime \prime \prime}(1)+\frac{1}{12} V_{K}^{\prime \prime}(1)$ be the primitive integer-valued degree 3 finite type invariant of $K$. Here $V_{K}(t)$ is the Jones polynomial of $K$.

Theorem 1.3. Let $K$ be a genus one knot with $\Delta_{K}(t)=1$. If $\lambda\left(\Sigma_{2}(K)\right)-2 w_{3}(K) \not \equiv$ $0(\bmod 16)$, then $K$ satisfies the cosmetic crossing conjecture. 
The cosmetic crossing conjecture has been confirmed for several cases; 2-bridge knots [Tor, fibered knots [Kal], knots whose double branched coverings are L-spaces with square-free 1st homology [LiMo, and some satellite knots BaKa]. Except the last satellite cases and the unknot, all the knots mentioned so far, including knots treated in Theorem 1.1, has non-trivial Alexander polynomial.

Theorem 1.3 gives examples of non-satellite knots with trivial Alexander polynomial satisfying the cosmetic crossing conjecture. Let $K=P(p, q, r)$ be the pretzel knot for odd $p, q, r$. Obviously, as long as $K$ is non-trivial, $g(K)=1$. The Alexander polynomial of $K$ is

$$
\Delta_{K}(t)=\frac{p q+q r+r p+1}{4} t+\frac{-2 p q-2 q r-2 r p+1}{2}+\frac{p q+q r+r p+1}{4} t^{-1} .
$$

Hence, for example, the pretzel knot $P(4 k+1,4 k+3,-(2 k+1))$ has the trivial Alexander polynomial.

Corollary 1.4. If $k \equiv 1,2(\bmod 4)$, the pretzel knot $P(4 k+1,4 k+3,-(2 k+1))$ satisfies the cosmetic crossing conjecture.

\section{Cosmetic Crossing of Genus one knot and Seifert surface}

We review an argument of [BFKP, Section 2, Section 3] that relates a cosmetic crossing change and Seifert matrix.

A crossing disk $D$ of an oriented knot $K$ is an embedded disk having exactly one positive and one negative crossing with $K$. A crossing change can be seen as $\varepsilon= \pm 1$ Dehn surgery on $\partial D$ for an appropriate crossing disk $D$, and the crossing is nugatory if and only if $\partial D$ bounds an embedded disk in $S^{3} \backslash K$.

Assume that $K$ admits a cosmetic crossing with the crossing disk $D$. Then as is discussed in BFKP, Section 2], there is a minimum genus Seifert surface $S$ of $K$ such that $\alpha:=D \cap S$ is a properly embedded, essential arc in $S$.

If $g(S)=1$, such an arc $\alpha$ is non-separating. We take simple closed curves $a_{x}, a_{y}$ of $S$ so that

- $a_{x}$ intersects $\alpha$ exactly once.

- $a_{x}$ and $a_{y}$ form a symplectic basis of $H_{1}(S ; \mathbb{Z})$.

Then we view $K=\partial S$ as a neighborhood of $a_{x} \cup a_{y}$ and express $K$ by a framed 2 -tangle $T$ as depicted in Figure 1.

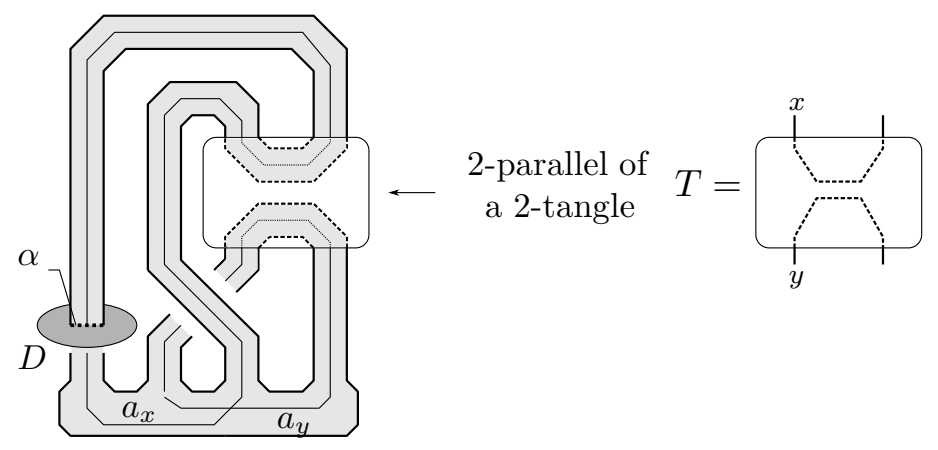

FIGURE 1. A spine tangle $T$ adapted to the cosmetic crossing 
We call the framed tangle $T$ a spine tangle of $K$ adapted to the cosmetic crossing of a genus one knot $K$. Let $M=\left(\begin{array}{cc}n & \ell \\ \ell & m\end{array}\right)$ be the linking matrix of $T$, where $n$ (resp. $m$ ) is the framing of the strand $x$ (resp. $y$ ) and $\ell$ is the linking number of two strands of $T$.

Let $K^{\prime}$ be a knot obtained from $K$ by crossing change along the crossing disk $D$. Then $S$ gives rise to a Seifert surface $S^{\prime}$ of $K^{\prime}$ ([BFKP Proposition 2.1]). $K^{\prime}$ has a spine tangle presentation $T^{\prime}$, so that $T^{\prime}$ and $T$ are the same as unframed tangles, and that the linking matrix of $T^{\prime}$ is $M^{\prime}=\left(\begin{array}{cc}n \pm 1 & \ell \\ \ell & m\end{array}\right)$

With respect to the basis $\left\{a_{x}, a_{y}\right\}$, the Seifert matrix $V$ of $K$ and the Seifert matrix $V^{\prime}$ of $K^{\prime}$ are given by

$$
V=\left(\begin{array}{cc}
n & \ell \\
\ell \pm 1 & m
\end{array}\right), \quad V^{\prime}=\left(\begin{array}{cc}
n \pm 1 & \ell \\
\ell \pm 1 & m
\end{array}\right)
$$

respectively. Since $K$ and $K^{\prime}$ are the same knot,

$$
\Delta_{K}(t) \doteq \operatorname{det}\left(V-t V^{T}\right) \doteq \operatorname{det}\left(V^{\prime}-t V^{\prime T}\right) \doteq \Delta_{K^{\prime}}(t) .
$$

By direct computation, this implies that

$$
m=0 .
$$

In particular, $K$ is algebraically slice.

\section{2-LOOP POLYNOMIAL OF GENUS ONE KNOT}

Here we quickly review the 2-loop polynomial. For details, see Oht. Let $\mathcal{B}$ be the space of open Jacobi diagram. For a knot $K$ in $S^{3}$, let $Z^{\sigma}(K) \in \mathcal{B}$ be the Kontsevich invariant of $K$, viewed so that it takes value in $\mathcal{B}$ by composing the inverse of the Poincaré-Birkoff-Witt isomorphism $\sigma: \mathcal{A}\left(S^{1}\right) \rightarrow \mathcal{B}$.

A Jacobi diagram whose edge is labeled by a power series $f(\hbar)=c_{0}+c_{1} \hbar+$ $c_{2} \hbar^{2}+c_{3} \hbar^{3}+\cdots$ represents the Jacobi diagram

$$
\left.\right|^{f(\hbar)}=c_{0}\left|+c_{1}\right|+c_{2} \underline{E}+c_{3} \underline{E}+\cdots
$$

It is known that (the logarithm of) the Kontsevich invariant $Z^{\sigma}(K)$ is written in the following form $\mathrm{GaKr}$, Kril.

$$
\begin{aligned}
\log _{\sqcup} Z^{\sigma}(K)=\overbrace{i: \text { finite }}^{\frac{1}{2} \log \left(\frac{\sinh (\hbar / 2)}{\hbar / 2}\right)-\frac{1}{2} \log \left(\Delta_{K}\left(e^{\hbar}\right)\right)} \\
+((\ell>2) \text {-loop parts }) .
\end{aligned}
$$

Here

- $\Delta_{K}(t)$ is the Alexander polynomial of $K$, normalized so that $\Delta_{K}(1)=1$ and $\Delta_{K}(t)=\Delta_{K}\left(t^{-1}\right)$ hold.

- $\log _{\sqcup}$ is the logarithm with respect to the disjoint union product $\sqcup$ of $\mathcal{B}$, given by

$$
\log _{\sqcup}(1+D)=D-\frac{1}{2} D \sqcup D+\frac{1}{3} D \sqcup D \sqcup D+\cdots .
$$


- $p_{i, j}\left(e^{\hbar}\right)$ is a polynomial of $e^{\hbar}$.

Let

$$
\Theta\left(t_{1}, t_{2}, t_{3} ; K\right)=\sum_{\varepsilon \in\{ \pm 1\}} \sum_{\sigma \in S_{3}} p_{i, 1}\left(t_{\sigma(1)}^{\varepsilon}\right) p_{i, 2}\left(t_{\sigma(2)}^{\varepsilon}\right) p_{i, 3}\left(t_{\sigma(3)}^{\varepsilon}\right) .
$$

Here $S_{3}$ is the symmetric group of degree 3. The 2-loop polynomial $\Theta_{K}\left(t_{1}, t_{2}\right) \in$ $\mathbb{Q}\left[t_{1}^{ \pm 1}, t_{2}^{ \pm 1}\right]$ of a knot $K$ is defined by

$$
\Theta_{K}\left(t_{1}, t_{2}\right)=\left.\Theta\left(t_{1}, t_{2}, t_{3} ; K\right)\right|_{t_{3}=t_{1}^{-1} t_{2}^{-1}} .
$$

The reduced 2-loop polynomial is a reduction of the 2-loop polynomial defined by

$$
\widehat{\Theta}_{K}(t)=\frac{\Theta_{K}(t, 1)}{\left(t^{\frac{1}{2}}-t^{-\frac{1}{2}}\right)^{2}} \in \mathbb{Q}\left[t^{ \pm 1}\right] .
$$

In general, although Ohtsuki developed fundamental techniques and machineries that enable us to compute $\Theta_{K}\left(t_{1}, t_{2}\right)$, the computation of the 2-loop polynomial is much more complicated than the computation of the 1-loop part (i.e., the Alexander polynomial). Fortunately, when the knot has genus one, Ohtsuki proved a direct formula of $\Theta_{K}\left(t_{1}, t_{2}\right)$ [Oht, Theorem 3.1]. Consequently he gave the following formula of the reduced 2-loop polynomial of genus one knots.

Theorem 3.1. Oht, Corollary 3.5] Let $K$ be a genus one knot expressed by using a framed 2-tangle $T$ as in Figure 1, and let $M=\left(\begin{array}{cc}n & \ell \\ \ell & m\end{array}\right)$ be the linking matrix of T. Then

$$
\begin{aligned}
\widehat{\Theta}_{K}(t)= & \left((n+m)\left(d-\frac{n m}{2}\right)-\ell\left(\ell+\frac{1}{2}\right)(\ell+1)+12 v_{3}\right)\left(-2-\frac{2 d+1}{3}\left(t+t^{-1}-2\right)\right) \\
& -4\left(m v_{2}^{x x}+n v_{2}^{y y}-\left(\ell+\frac{1}{2}\right) v_{2}^{x y}+3 v_{3}\right) \Delta_{K}(t)
\end{aligned}
$$

Here

- $d=n m-\ell^{2}-\ell$. In particular, $\Delta_{K}(t)=d t+(1-2 d)+d t^{-1}$.

- $v_{2}^{x x}, v_{2}^{y y}, v_{2}^{x y}$ (resp. $v_{3}$ ) are some integer-valued finite type invariant of $T$ whose degree is 2 (resp. 3), which do not depend on the framing.

\section{Constraint For COSmetic CROssings}

We prove the Theorem 1.2 and Theorem 1.3 at the same time.

Theorem 4.1. Let $K$ be a genus one knot. If $K$ admits a cosmetic crossing, then $\Delta_{K}(t)=1$ and $\lambda\left(\Sigma_{2}(K)\right)-2 w_{3}(K) \equiv 0(\bmod 16)$.

Proof. Assume that $K$ is a genus one knot admitting a cosmetic crossing. We express $K$ using a spine tangle $T$ adapted to the cosmetic crossing. Then as we have seen (2.1), the linking matrix of $T$ is $M=\left(\begin{array}{ll}n & \ell \\ \ell & 0\end{array}\right)$. Moreover, for the knot $K^{\prime}$ obtained by the crossing change, $K^{\prime}$ has a spine tangle $T^{\prime}$ which is identical with $T$ as an unframed tangle with linking matrix is $M^{\prime}=\left(\begin{array}{cc}n \pm 1 & \ell \\ \ell & 0\end{array}\right)$. 
Since the finite type invariants $v_{2}^{x x}, v_{2}^{y y}, v_{2}^{x y}$ and $v_{3}$ do not depend on the framing, by Theorem 3.1 .

$$
\begin{aligned}
0 & =\widehat{\Theta}_{K}(t)-\widehat{\Theta}_{K^{\prime}}(t) \\
& =d\left(-2-\frac{2 d+1}{3}\left(t+t^{-1}-2\right)\right)-4 v_{2}^{y y}\left(d t+(1-2 d)+d t^{-1}\right) \\
& =d\left(-\frac{2 d+1}{3}-4 v_{2}^{y y}\right) t+\frac{d(4 d-4)}{3}+4 v_{2}^{y y}(2 d-1)+d\left(-\frac{2 d+1}{3}-4 v_{2}^{y y}\right) t^{-1} .
\end{aligned}
$$

Therefore

$$
d\left(-\frac{2 d+1}{3}-4 v_{2}^{y y}\right)=\frac{d(4 d-4)}{3}+4 v_{2}^{y y}(2 d-1)=0 .
$$

If $d \neq 0$, by (4.1) $d=\frac{1}{4}$. Since $d \in \mathbb{Z}$, this is a contradiction so we conclude $d=0$ and $\Delta_{K}(t)=1$.

Then by (4.1), $d=0$ implies $v_{2}^{y y}=0$. Moreover, since $d=n m-\ell^{2}-\ell=-\ell(\ell+1)$, we get $\ell=0,-1$. Thus by Theorem 3.1, the reduced 2-loop polynomial is

$$
\widehat{\Theta}_{K}(t)=12 v_{3}\left(-2-\frac{1}{3}\left(t+t^{-1}-2\right)\right)-4\left(-\left(\ell+\frac{1}{2}\right) v_{2}^{x y}-3 v_{3}\right)
$$

hence

$$
\widehat{\Theta}_{K}(1)=-12 v_{3}+4\left(\ell+\frac{1}{2}\right) v_{2}^{x y}, \widehat{\Theta}_{K}(-1)=4 v_{3}+4\left(\ell+\frac{1}{2}\right) v_{2}^{x y} .
$$

On the other hand, by [Oht, Proposition 1.1]

$$
\widehat{\Theta}_{K}(1)=2 w_{3}(K), \quad \widehat{\Theta}_{K}(-1)=-\frac{1}{12} V_{K}^{\prime}(-1) V_{K}(-1) .
$$

Since $\Delta_{K}(-1)=V_{K}(-1)=1$, by Mullins' formula of the Casson-Walker invariant $\lambda_{w}$ of the double branched coverings $\mathrm{Mul}$,

$$
\lambda_{w}\left(\Sigma_{2}(K)\right)=-\frac{V_{K}^{\prime}(-1)}{6 V_{K}(-1)}+\frac{\sigma(K)}{4}
$$

we get

$$
\widehat{\Theta}_{K}(-1)=\frac{1}{2} \lambda_{w}\left(\Sigma_{2}(K)\right)
$$

For an integral homology sphere, the Casson invariant $\lambda$ is twice of the CassonWalker invariant $\lambda_{w}$ hence we conclude

$$
\lambda\left(\Sigma_{2}(K)\right)-2 w_{3}(K)=\widehat{\Theta}_{K}(-1)-\widehat{\Theta}_{K}(1)=16 v_{3} .
$$

Proof of Corollary 1.4. The reduced 2-loop polynomial of genus pretzel knots $P(p, q, r)$ was given in [Oht, Example 3.6]. In particular, for $K=P(4 k+1,4 k+3,-(2 k+1))$, $\widehat{\Theta}_{K}(1)$ and $\widehat{\Theta}_{K}(-1)$ are given by

$$
\widehat{\Theta}_{K}(1)=-\frac{1}{8}(4 k+2)(4 k+4)(-2 k), \widehat{\Theta}_{K}(-1)=-\frac{1}{24}(4 k+2)(4 k+4)(-2 k)
$$

hence

$$
\begin{aligned}
\lambda\left(\Sigma_{2}(K)\right)-2 w_{3}(K) & =\widehat{\Theta}_{K}(-1)-\widehat{\Theta}_{K}(1)=\frac{1}{12}(4 k+2)(4 k+4)(-2 k) \\
& =-16 \frac{(2 k+1)(k+1) k}{12} .
\end{aligned}
$$


When $k \equiv 1,2(\bmod 4), \frac{(2 k+1)(k+1) k}{12} \notin \mathbb{Z}$ hence $K$ does not admit cosmetic crossing by Theorem 1.3 .

\section{ACKNOWLEDGEMENT}

The author has been partially supported by JSPS KAKENHI Grant Number 19K03490,16H02145.

\section{REFERENCES}

[BFKP] C. Balm, S. Friedl, E. Kalfagianni, and M. Powell, Cosmetic crossings and Seifert matrices, Comm. Anal. Geom. 20 (2012), 235-253.

[BaKa] C. Balm and E. Kalfagianni, Knots without cosmetic crossings, Topology Appl. 207 (2016), 33-42.

[GaKr] S. Garoufalidis and A. Kricker, A rational noncommutative invariant of boundary links, Geom. Topol. 8 (2004), 115-204.

[Kal] E. Kalfagianni, Cosmetic crossing changes of fibered knots, J. reine angew. Math. 669 (2012), 151-164.

[Kir] R. Kirby (ed). Problems in low-dimensional topology, Edited by Rob Kirby. AMS/IP Stud. Adv. Math., 2.2, Geometric topology (Athens, GA, 1993), 3-473, Amer. Math. Soc., Providence, RI, 1997.

[Kri] A. Kricker, The lines of the Kontsevich integral and Rozansky's rationality conjecture, arXiv:math/0005284

[LiMo] T. Lidman and A. Moore, Cosmetic surgery in L-spaces and nugatory crossings, Trans. Amer, Math. Soc. 369 (2017), 3639-3654.

[Mul] D. Mullins, The generalized Casson invariant for 2-fold branched covers of $S^{3}$ and the Jones polynomial, Topology 32 (1993), 419-438.

[Oht] T. Ohtsuki, On the 2-loop polynomial of knots, Geom. Topol. 11 (2007), 1357-1475.

[Tor] I. Torisu, On nugatory crossings for knots, Topology Appl. 92 (1999), 119-129. 